\title{
Fractional-Order Derivative Model of Rift Valley Fever in Urban Peridomestic Cycle
}

\author{
Saul C. Mpeshe $\mathbb{D}$ \\ Department of Mathematical Sciences, University of Iringa, P. O. Box 200, Iringa, Tanzania \\ Correspondence should be addressed to Saul C. Mpeshe; saul.mpeshe@uoi.ac.tz
}

Received 12 April 2021; Revised 7 May 2021; Accepted 20 May 2021; Published 28 May 2021

Academic Editor: Abdul Qadeer Khan

Copyright (c) 2021 Saul C. Mpeshe. This is an open access article distributed under the Creative Commons Attribution License, which permits unrestricted use, distribution, and reproduction in any medium, provided the original work is properly cited.

Rift Valley fever is a zoonotic disease which is mainly transmitted by mosquitoes and has potential to affect humans and animals. To gain some understanding on its dynamics in an urban peridomestic cycle, a fractional-order derivative model is formulated and analysed. The basic reproduction number $\mathscr{R}_{0}$ is computed and used in analysing the stability of disease when an outbreak occurs. Numerical simulations are performed in order to the variation of each population at order $\alpha=1,0.75,0.5$, and 0.25 . Results from simulations show that there is an increase in susceptible and exposed population in both human and mosquitoes as the value of $\alpha$ decreases. The infected population decreases with a decrease in the value of $\alpha$. However, a rapid increase in susceptible mosquitoes is observed just after the first 30 days and a rapid decrease in infected human and mosquitoes after the first 30 days for $\alpha=1$. Hence, fractional-order derivative also plays a significant role in providing insight on disease transmission and dynamics.

\section{Introduction}

Rift Valley fever (RVF) is a mosquito-borne zoonotic viral disease which affects both humans and animals. The main causative agent of RVF is the Rift Valley fever virus (RVFV) of the genus Phlebovirus in the family Bunyaviridae [1]. The virus was first isolated in sheep in 1930 during an outbreak in Kenya [2].

Domestic animals are highly susceptible to RVFV infection and are considered to be the major amplifiers of the virus [3]. Humans are also at a high risk to contract RVFV infection, and they have the ability to develop sufficient viraemia to infect mosquitoes [3].

The transmission of RVFV to humans can result through different cycles: the sylvatic cycle and the urban peridomestic cycle [1]. In a sylvatic cycle, humans become infected mostly from contact with the blood or organs of infected animals, while, in the urban peridomestic cycle, humans become infected through the bites of mosquitoes [1]. The Egyptian outbreak of 1977-1978 is known to be peridomestic and anthropophilic [4]. There are possibilities also for human infections to result from other blood-feeding insects, which might be serve as mechanical transmitters of infection. The current urban peridomestic outbreaks of RVF include the 2019 outbreak in Sudan where 1,129 case patients and 19 deaths were reported [5] and that of Mayotte (France) where 143 human cases were reported [6].

\section{The RVF Model}

Mathematical modelling has played a major role in understanding better the global behaviour of infectious diseases. Modelling of infectious diseases using ordinary differential equations (ODEs) also known as integer-order differential equations (IDEs) has proven to be valuable in analysing the dynamics of various diseases including Rift Valley fever [7-9]. However, ODE or IDE cannot store memory.

Memory is important in the transmission dynamics and control of infectious diseases. The experience and/or knowledge we have about the spread of a certain disease in the past affects our response during an epidemic outbreak. Knowing the history of a certain disease in our area may help us to think of different control strategies. Therefore, it is crucial to incorporate memory in our epidemic models for a better understanding of the disease dynamics and control. 
The best way to do this is to use fractional-order derivative models.

Fractional-order derivatives are a generalization of the integer-order derivative and integrals to an arbitrary real or complex order. They are powerful mathematical tools which can be used to model the real-world phenomenon which involve memory, wide range of interactions, and hereditary properties, a property which is neglected by integer-order derivatives [10].

Modelling with fractional-order derivatives has motivated many people involved in research in science including epidemiology. This is evident from the work by Silva and Torres [11], Boukhouima et al. [10], Ghanbari et al. [12], Alkahtani and Alzaid [13], Tuan et al. [14], and Khan and Atangana [15], just to mention a few. Motivated by their work, a fractional-order derivative model of RVF infection in Caputo derivative is presented in this paper. Other works involving Caputo derivative include works of Ndaïrou et al. [16], Aba Oud et al. [17], and Chu et al. [18] just to mention a few. The paper begins with the formulation of a model using ODEs, and then the model is converted to a fractional-order derivative model using the Caputo derivative. The global stability analysis of the model is established using suitable construction of fractional Lyapunov function, and numerical simulation is done using the predictor-corrector method as outlined by Diethelm et al. [19].

2.1. Model Formulation. The model formulation considers two populations, namely: humans and mosquitoes. There is natural death rate in each stage because the infection may take long time, and therefore individual may die naturally. The mode of transmission considered in this model is from mosquito to human and human to mosquito as shown in Figure 1. The mosquito population is divided into three compartments, namely: susceptible $S_{m}$, exposed $E_{m}$, and infectious $I_{m}$. The total mosquito population is given by $N_{m}=S_{m}+E_{m}+I_{m}$. Mosquitoes are also capable of transmitting the virus directly to their offspring (vertical transmission) via eggs leading to new generations of infected mosquitoes hatching from eggs. Therefore, we include the vertical transmission rate parameter $q$ in the model. The human population has four compartments, namely, susceptible $S_{h}$, exposed $E_{h}$, infectious $I_{h}$, and recovered $R_{h}$. The total human population is given by $N_{h}=S_{h}+E_{h}+I_{h}+R_{h}$.

The parameters and their description as they have been used in this paper are presented in Table 1.

Figure 1 represents the flow of diagram of RVF infection in each population involved.

From Table 1 and Figure 1, an SEIR model is formulated using first-order nonlinear ordinary differential equations as follows:

$$
\begin{aligned}
& \frac{\mathrm{d} S_{h}}{\mathrm{~d} t}=b_{h} N_{h}-\mu_{h} S_{h}-\lambda_{m h} \frac{I_{m}}{N_{m}} S_{h}, \\
& \frac{\mathrm{d} E_{h}}{\mathrm{~d} t}=\lambda_{m h} \frac{I_{m}}{N_{m}} S_{h}-\left(\varepsilon_{h}+\mu_{h}\right) E_{h},
\end{aligned}
$$

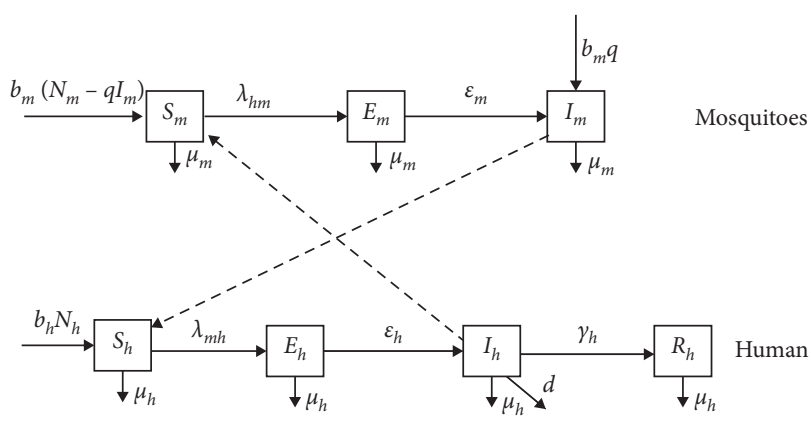

Figure 1: Transmission diagram for RVF.

TABle 1: Description of parameters.

\begin{tabular}{lc}
\hline Parameter & Description \\
\hline$b_{m}$ & Birth rate in mosquitoes \\
$b_{h}$ & Birth rate in human \\
$\lambda_{h m}$ & Adequate contact rate: human to mosquito \\
$\lambda_{m h}$ & Adequate contact rate: mosquito to human \\
$\varepsilon_{m}$ & Rate of progression from latency to infectious \\
$\varepsilon_{h}$ & Rate of progression from latency to infectious human \\
$\mu_{m}$ & Natural death rate of mosquitoes \\
$\mu_{h}$ & Natural death rate of human \\
$q$ & Rate of vertical transmission \\
$d$ & Disease-induced death rate in human \\
$\gamma_{h}$ & Disease recovery rate in human \\
\hline
\end{tabular}

$$
\begin{aligned}
& \frac{\mathrm{d} I_{h}}{\mathrm{~d} t}=\varepsilon_{h} E_{h}-\left(\mu_{h}+\gamma_{h}+d\right) I_{h}, \\
& \frac{\mathrm{d} R_{h}}{\mathrm{~d} t}=\gamma_{h} I_{h}-\mu_{h} R_{h}, \\
& \frac{\mathrm{d} S_{m}}{\mathrm{~d} t}=b_{m}\left(N_{m}-q I_{m}\right)-\mu_{m} S_{m}-\lambda_{h m} \frac{I_{h}}{N_{h}} S_{m}, \\
& \frac{\mathrm{d} E_{m}}{\mathrm{~d} t}=\lambda_{h m} \frac{I_{h}}{N_{h}} S_{m}-\left(\varepsilon_{m}+\mu_{m}\right) E_{m}, \\
& \frac{\mathrm{d} I_{m}}{\mathrm{~d} t}=\varepsilon_{m} E_{m}-\left(\mu_{m}-b_{m} q\right) I_{m} .
\end{aligned}
$$

Definition 1. Let $f(t)$ be a continuous function differentiable $n$-times. The Caputo fractional derivative of $f(t)$ is defined as in the study by Petras [20] as follows:

$$
{ }_{a}^{C} D_{t}^{\alpha} f(t)=\frac{1}{\Gamma(n-\alpha)} \int_{a}^{t} \frac{f^{(n)}(\tau)}{(t-\tau)^{\alpha-n+1}} \mathrm{~d} \tau,
$$

for $n-1<\alpha<n$.

For $0<\alpha \leq 1$ and $a=0$, the definition can be written as in [16] as

$$
{ }_{0}^{C} D_{t}^{\alpha} f(t)=\frac{1}{\Gamma(1-\alpha)} \int_{0}^{t} \frac{f^{\prime}(\tau)}{(t-\tau)^{\alpha}} \mathrm{d} \tau .
$$


Definition 2. The integral corresponding to Caputo fractional derivative is defined as in [17] as

$$
I_{t}^{\alpha} f(t)=\frac{1}{\Gamma(\alpha)} \int_{0}^{t} \frac{f(\tau)}{(t-\tau)^{\alpha-1}} \mathrm{~d} \tau
$$

for $\alpha \in(0,1), t>0$.

The fractional-order derivative model using Caputo derivative is now defined as

$$
\begin{aligned}
& { }_{0}^{C} D_{t}^{\alpha} S_{h}=b_{h} N_{h}-\mu_{h} S_{h}-\lambda_{m h} \frac{I_{m}}{N_{m}} S_{h}, \\
& { }_{0}^{C} D_{t}^{\alpha} E_{h}=\lambda_{m h} \frac{I_{m}}{N_{m}} S_{h}-\left(\varepsilon_{h}+\mu_{h}\right) E_{h}, \\
& { }_{0}^{C} D_{t}^{\alpha} I_{h}=\varepsilon_{h} E_{h}-\left(\mu_{h}+\gamma_{h}+d\right) I_{h}, \\
& { }_{0}^{C} D_{t}^{\alpha} R_{h}=\gamma_{h} I_{h}-\mu_{h} R_{h}, \\
& { }_{0}^{C} D_{t}^{\alpha} S_{m}=b_{m}\left(N_{m}-q I_{m}\right)-\mu_{m} S_{m}-\lambda_{h m} \frac{I_{h}}{N_{h}} S_{m}, \\
& { }_{0}^{C} D_{t}^{\alpha} E_{m}=\lambda_{h m} \frac{I_{h}}{N_{h}} S_{m}-\left(\varepsilon_{m}+\mu_{m}\right) E_{m}, \\
& { }_{0}^{C} D_{t}^{\alpha} I_{m}=\varepsilon_{m} E_{m}-\left(\mu_{m}-b_{m} q\right) I_{m},
\end{aligned}
$$

where ${ }_{0}^{C} D_{t}^{\alpha}$ is the Caputo derivative of order $\alpha \in(0,1)$, with initial conditions as follows:

$$
\begin{gathered}
S_{h}(0)=S_{h 0} \geq 0, \\
E_{h}(0)=E_{h 0} \geq 0, \\
I_{h}(0)=I_{h 0} \geq 0, \\
R_{h}(0)=R_{h 0} \geq 0, \\
S_{h}(0)=S_{m 0} \geq 0, \\
E_{m}(0)=E_{m 0} \geq 0, \\
I_{m}(0)=I_{m 0} \geq 0 .
\end{gathered}
$$

The order $\alpha$ indicates the index of memory in the system. Let

$$
\begin{aligned}
& s_{h}=\frac{S_{h}}{N_{h}}, e_{h}=\frac{E_{h}}{N_{h}}, i_{h}=\frac{I_{h}}{N_{h}}, r_{h}=\frac{R_{h}}{N_{h}}, \\
& s_{m}=\frac{S_{m}}{N_{m}}, e_{m}=\frac{E_{m}}{N_{m}}, i_{m}=\frac{I_{m}}{N_{m}},
\end{aligned}
$$

where $s_{h}, e_{h}, i_{h}, r_{h}, s_{m}, e_{m}, i_{m}$ are population proportions. Then, $s_{h}+e_{h}+i_{h}+r_{h}=1$ and $s_{m}+e_{m}+i_{m}=1$. Since RVF outbreak usually occurs over a short period, then $N_{h}$ and $N_{m}$ can be considered to be constant at the time of the outbreak. Therefore, ${ }_{0}^{C} D_{t}^{\alpha} x_{i}=\left(1 / N_{h}\right)_{0}^{C} D_{t}^{\alpha} X_{i}$ for $x_{i}=\left(s_{h}, e_{h}, i_{h}, r_{h}\right)$ and $X_{i}=\left(S_{h}, E_{h}, I_{h}, R_{h}\right)$ and ${ }_{0}^{C} D_{t}^{\alpha} x_{j}=\left(1 / N_{h}\right)_{0}^{C} D_{t}^{\alpha} X_{j}$ for $x_{j}=\left(s_{m}, e_{m}, i_{m}\right)$ and $X_{j}=\left(S_{m}, E_{m}, I_{m}\right)$. Thus, the model system $(5 \mathrm{a})-(5 \mathrm{~g})$ can now be rescaled to

$$
\begin{aligned}
& { }_{0}^{C} D_{t}^{\alpha} s_{h}=b_{h}-\mu_{h} s_{h}-\lambda_{m h} i_{m} s_{h}, \\
& { }_{0}^{C} D_{t}^{\alpha} e_{h}=\lambda_{m h} i_{m} s_{h}-\left(\varepsilon_{h}+\mu_{h}\right) e_{h}, \\
& { }_{0}^{C} D_{t}^{\alpha} i_{h}=\varepsilon_{h} e_{h}-\left(\mu_{h}+\gamma_{h}+d\right) i_{h}, \\
& { }_{0}^{C} D_{t}^{\alpha} r_{h}=\gamma_{h} i_{h}-\mu_{h} r_{h}, \\
& { }_{0}^{C} D_{t}^{\alpha} s_{m}=b_{m}\left(1-q i_{m}\right)-\mu_{m} s_{m}-\lambda_{h m} i_{h} s_{m}, \\
& { }_{0}^{C} D_{t}^{\alpha} e_{m}=\lambda_{h m} i_{h} s_{m}-\left(\varepsilon_{m}+\mu_{m}\right) e_{m}, \\
& { }_{0}^{C} D_{t}^{\alpha} i_{m}=\varepsilon_{m} e_{m}-\left(\mu_{m}-b_{m} q\right) i_{m} .
\end{aligned}
$$

Since $r_{h}$ is not involved in deriving other equations, then the equation for $r_{h}$ is omitted from the analysis for its value which can be calculated from the values of $s_{h}, e_{h}$, and $i_{h}$. Thus, the model system is reduced to

$$
\begin{aligned}
& { }_{0}^{C} D_{t}^{\alpha} s_{h}=b_{h}-\mu_{h} s_{h}-\lambda_{m h} i_{m} s_{h}, \\
& { }_{0}^{C} D_{t}^{\alpha} e_{h}=\lambda_{m h} i_{m} s_{h}-\left(\varepsilon_{h}+\mu_{h}\right) e_{h}, \\
& { }_{0}^{C} D_{t}^{\alpha} i_{h}=\varepsilon_{h} e_{h}-\left(\mu_{h}+\gamma_{h}+d\right) i_{h}, \\
& { }_{0}^{C} D_{t}^{\alpha} s_{m}=b_{m}\left(1-q i_{m}\right)-\mu_{m} s_{m}-\lambda_{h m} i_{h} s_{m}, \\
& { }_{0}^{C} D_{t}^{\alpha} e_{m}=\lambda_{h m} i_{h} s_{m}-\left(\varepsilon_{m}+\mu_{m}\right) e_{m}, \\
& { }_{0}^{C} D_{t}^{\alpha} i_{m}=\varepsilon_{m} e_{m}-\left(\mu_{m}-b_{m} q\right) i_{m} .
\end{aligned}
$$

2.2. Feasibility of the Model Solution. To show that the model solution exists and is in the positive region $\mathbb{R}_{+}^{6}$, we show that ${ }_{0}^{C} D_{t}^{\alpha} x_{i} \geq 0$ in the region $\mathbb{R}_{+}^{6}$. Using the model system (9a)-(9f), we have

$$
\begin{aligned}
& \left.{ }_{0}^{C} D_{t}^{\alpha} s_{h}\right|_{s_{h}=0}=b_{h}>0, \\
& \left.{ }_{0}^{C} D_{t}^{\alpha} e_{h}\right|_{e_{h}=0}=\lambda_{m h} i_{m} s_{h} \geq 0, \\
& \left.{ }_{0}^{C} D_{t}^{\alpha} i_{h}\right|_{i_{h}=0}=\varepsilon_{h} e_{h} \geq 0, \\
& \left.{ }_{0}^{C} D_{t}^{\alpha} s_{m}\right|_{s_{m}=0}=b_{m}\left(1-q i_{m}\right)>0, \\
& \left.{ }_{0}^{C} D_{t}^{\alpha} e_{m}\right|_{e_{m}=0}=\lambda_{h m} i_{h} s_{m} \geq 0, \\
& \left.{ }_{0}^{C} D_{t}^{\alpha} i_{m}\right|_{i_{m}=0}=\varepsilon_{m} e_{m} \geq 0 .
\end{aligned}
$$

Hence, the model solution is feasible and positive in $\Omega=\left(s_{h}, e_{h}, i_{h}, s_{m}, e_{m}, i_{m}\right) \geq 0 \in \mathbb{R}_{+}^{6}$.

To show the solution is positively invariant in the feasible region, we show that $s_{h}(t)+e_{h}(t)+i_{h}(t) \leq\left(b_{h} / \mu_{h}\right)$ and $s_{m}(t)+e_{m}(t)+i_{m}(t) \leq\left(b_{m} / \mu_{m}\right) \quad$ as $t \longrightarrow \infty$. Since $s_{h}+e_{h}+i_{h}+r_{h}=1$, it follows that 


$$
{ }_{0}^{C} D_{t}^{\alpha}\left(s_{h}+e_{h}+i_{h}\right) \leq b_{h}-\mu_{h}\left(s_{h}+e_{h}+i_{h}\right) .
$$

Solving this inequality gives

$$
s_{h}(t)+e_{h}(t)+i_{h}(t) \leq\left(s_{h}(0)+e_{h}(0)+i_{h}(0)\right) E_{\alpha}\left(-\mu_{h} t^{\alpha}\right)+\frac{b_{h}}{\mu_{h}}\left(1-E_{\alpha}\left(-\mu_{h} t^{\alpha}\right)\right)
$$

where $E_{\alpha}(\cdot)$ is a Mittag-Leffler operator.

As $t \longrightarrow \infty$, we have

$$
s_{h}(t)+e_{h}(t)+i_{h}(t) \leq \frac{b_{h}}{\mu_{h}} .
$$

Similarly,

$$
{ }_{0}^{C} D_{t}^{\alpha}\left(s_{m}+e_{m}+i_{m}\right) \leq b_{m}-\mu_{m}\left(s_{m}+e_{m}+i_{m}\right) .
$$

Solving this equation gives

$$
s_{m}(t)+e_{m}(t)+i_{m}(t) \leq\left(s_{m}(0)+e_{m}(0)+i_{m}(0)\right) E_{\alpha}\left(-\mu_{m} t^{\alpha}\right)+\frac{b_{m}}{\mu_{m}}\left(1-E_{\alpha}\left(-\mu_{m} t^{\alpha}\right)\right)
$$

where also $E_{\alpha}(\cdot)$ is a Mittag-Leffler operator.

As $t \longrightarrow \infty$, we have

$$
s_{m}(t)+e_{m}(t)+i_{m}(t) \leq \frac{b_{m}}{\mu_{m}} .
$$

Hence, the model solution is positively invariant in $\mathbb{R}_{+}^{6}$. This means that the model solution will remain in the feasible region $\Omega$ if it starts in $\Omega$.
2.3. The Basic Reproduction Number. The computation of basic reproduction number $\mathscr{R}_{0}$ is done by applying the next generation method as described by van den Driessche and Watmough [21]. From the model system (9a)-(9f), it is found that

$$
\begin{aligned}
& F=\left[\begin{array}{cccc}
0 & 0 & 0 & \lambda_{m h} s_{h}^{*} \\
0 & 0 & 0 & 0 \\
0 & \lambda_{h m} s_{m}^{*} & 0 & 0 \\
0 & 0 & 0 & 0
\end{array}\right] \\
& V=\left[\begin{array}{cccc}
\varepsilon_{h}+\mu_{h} & 0 & 0 & 0 \\
-\varepsilon_{h} & \mu_{h}+\gamma_{h}+d & 0 & 0 \\
0 & 0 & \varepsilon_{m}+\mu_{m} & 0 \\
0 & 0 & -\varepsilon_{m} & \mu_{m}-b_{m} q
\end{array}\right] \text {, } \\
& F V^{-1}=\left[\begin{array}{cccc}
0 & 0 & \frac{\varepsilon_{m} \lambda_{m h} s_{h}^{*}}{\left(\varepsilon_{m}+\mu_{m}\right)\left(\mu_{m}-b_{m} q\right)} \frac{\lambda_{m h} s_{h}^{*}}{\mu_{m}-b_{m} q} \\
0 & 0 & 0 & 0 \\
\frac{\varepsilon_{h} \lambda_{h m} s_{m}^{*}}{\left(\varepsilon_{h}+\mu_{h}\right)\left(\mu_{h}+\gamma_{h}+d\right)} \frac{\lambda_{h m} s_{m}^{*}}{\mu_{h}+\gamma_{h}+d} & 0 & 0 \\
0 & 0 & 0 & 0
\end{array}\right] .
\end{aligned}
$$


The largest eigenvalue of the $F V^{-1}$ gives the expression for the basic reproduction number $\mathscr{R}_{0}$. Computing the eigenvalues of $F V^{-1}$ and substituting $s_{h}^{*}=\left(b_{h} / \mu_{h}\right)$ and $s_{m}^{*}=$ $\left(b_{m} / \mu_{m}\right)$ at disease-free equilibrium give

$$
\mathscr{R}_{0}=\sqrt{\frac{b_{h} b_{m} \varepsilon_{h} \varepsilon_{m} \lambda_{m h} \lambda_{h m}}{\mu_{h} \mu_{m}\left(\varepsilon_{h}+\mu_{h}\right)\left(\mu_{h}+\gamma_{h}+d\right)\left(\varepsilon_{m}+\mu_{m}\right)\left(\mu_{m}-b_{m} q\right)}},
$$

with $\mu_{m}-b_{m} q>0$. Notice that $\mu_{m}-b_{m} q>0=$ $\mu_{m}\left(1-\left(b_{m} q / \mu_{m}\right)\right)$ where the quantity $\left(b_{m} q / \mu_{m}\right)$ is the mean number of mosquitoes infected as a result of vertical transmission during its life span.

Observe that $\mathscr{R}_{0}$ has six components: the component $\left(b_{m} / \mu_{m}\right)$ represents the mean number of births of mosquitoes during its lifespan; $\left(b_{h} / \mu_{h}\right)$ represents the mean number of births of human during the lifespan; $\varepsilon_{h} /\left(\varepsilon_{h}+\mu_{h}\right)$ represents the probability that an infected human survives throughout the incubation period and becomes infectious; $\varepsilon_{m} /\left(\varepsilon_{m}+\mu_{m}\right)$ represents the probability that an infected mosquito survives throughout the incubation period and becomes infectious; $\lambda_{h m} /\left(\mu_{h}+\gamma_{h}+d\right)$ represents the mean number of mosquitoes infected by human during the infectious period, and $\lambda_{m h} /\left(\mu_{m}-b_{m} q\right)$ represents the mean number of human infected by mosquitoes during its infectious period.

\section{Stability Analysis of Equilibrium Points}

To compute the equilibrium points, the left-hand side of model system (9a)-(9f) is equated to zero. For the diseasefree equilibrium $P_{0}$, we have $e_{h}=i_{h}=e_{m}=i_{m}=0$, giving

$$
P_{0}=\left(\frac{b_{h}}{\mu_{h}}, 0,0, \frac{b_{m}}{\mu_{m}}, 0,0\right) \text {. }
$$

Further computation gives the endemic equilibrium $P^{*}$ as $P^{*}=\left(s_{h}^{*}, e_{h}^{*}, i_{h}^{*}, s_{m}^{*}, e_{m}^{*}, i_{m}^{*}\right)$ where

$$
\begin{aligned}
& s_{h}^{*}=\frac{b_{h}}{\mu_{h}+\lambda_{m h} i_{m}^{*}}, \\
& e_{h}^{*}=\frac{\lambda_{m h} i_{m}^{*} s_{h}^{*}}{\varepsilon_{h}+\mu_{h}}, \\
& i_{h}^{*}=\frac{\varepsilon_{h} e_{h}^{*}}{\mu_{h}+\gamma_{h}+d}, \\
& s_{m}^{*}=\frac{b_{m}\left(1-q i_{m}^{*}\right)}{\mu_{m}+\lambda_{h m} i_{h}^{*}}, \\
& e_{m}^{*}=\frac{\lambda_{h m} i_{h}^{*} s_{m}^{*}}{\varepsilon_{m}+\mu_{m}}, \\
& i_{m}^{*}=\frac{\varepsilon_{m} e_{m}^{*}}{\mu_{m}-b_{m} q} .
\end{aligned}
$$

\subsection{Local Stability of the Disease-Free Equilibrium}

Theorem 1. The disease-free equilibrium of the RVF models (9a)-(9f) is locally asymptotically stable if $\mathscr{R}_{0}<1$ and unstable if $\mathscr{R}_{0}>1$.

Proof. The theorem is proved by showing that all the eigenvalues of the Jacobian matrix $J\left(P_{0}\right)$ of the fractionalorder RVF model (9a)-(9f) evaluated at the disease-free equilibrium satisfy the condition $|\arg (\operatorname{eig}(J))|=$ $\left|\arg \left(\lambda_{i}\right)\right|>\alpha(\pi / 2)$. That is, the Jacobian matrix $J$ has negative real eigenvalues. From the model (9a)-(9f), the Jacobian matrix at $P_{0}$ is given by

$$
J\left(P_{0}\right)=\left[\begin{array}{cccccc}
-\mu_{a} & 0^{\prime} & 0 & 0 & 0 & -\lambda_{m h} s_{h}^{*} \\
0 & -\left(\varepsilon_{h}+\mu_{h}\right) & 0 & 0 & 0 & \lambda_{m h} s_{h}^{*} \\
0 & \varepsilon_{h} & -\left(\mu_{h}+\gamma_{h}+d\right) & 0 & 0 & 0 \\
0 & 0 & -\lambda_{h m} s_{m}^{*} & -\mu_{m} & 0 & -b_{m} q \\
0 & 0 & \lambda_{h m} s_{m}^{*} & 0 & -\left(\varepsilon_{m}+\mu_{m}\right) & 0 \\
0 & 0 & 0 & 0 & \varepsilon_{m} & -\left(\mu_{m}-b_{m} q\right)
\end{array}\right] .
$$

From $J\left(P_{0}\right)$, the diagonal entries $-\mu_{h}$ and $-\mu_{m}$ form the first two eigenvalues of the matrix. If the columns and their corresponding rows of these two eigenvalues are eliminated from the matrix, the remaining is a $4 \times 4$ matrix given by 


$$
J^{*}\left(P_{0}\right)=\left[\begin{array}{cccc}
-\left(\varepsilon_{h}+\mu_{h}\right) & 0 & 0 & \lambda_{m h} s_{h}^{*} \\
\varepsilon_{h} & -\left(\mu_{h}+\gamma_{h}+d\right) & 0 & 0 \\
0 & \lambda_{h m} s_{m}^{*} & -\left(\varepsilon_{m}+\mu_{m}\right) & 0 \\
0 & 0 & \varepsilon_{m} & -\left(\mu_{m}-b_{m} q\right)
\end{array}\right]
$$

It is easy to see that the remaining matrix $J^{*}\left(P_{0}\right)$ is a Metzler stable matrix whose eigenvalues are all negative. Hence, the Jacobian matrix $J\left(P_{0}\right)$ has all its eigenvalues negative, and thus the disease-free equilibrium is locally asymptotically stable.

\subsection{Global Stability of the Disease-Free Equilibrium}

Theorem 2. The disease-free equilibrium of the fractionalorder RVF model (9a)-(9f) is globally asymptotically stable if $\mathscr{R}_{0}<1$ and unstable if $\mathscr{R}_{0}>1$.

Proof. We apply the Lyapunov functional approach for fractional differential equations.

Definition 3. Let $V\left(x_{i}(t)\right)=\sum_{i}^{n} \omega_{i} \phi_{i}\left(x_{i}(t)\right)$ be a $C^{1}$ function defined on some domain in $\mathbb{R}_{+}^{n}$ and $x_{i}(t)$ be a solution of the model system ${ }_{0}^{C} D_{t}^{\alpha} x_{i}(t)=f\left(x_{i}(t)\right), x_{i}(0)=x_{0}, \alpha \in(0,1)$. Then, the Caputo derivative of $V$ along $x_{i}(t)$ is given by

$$
{ }_{0}^{C} D_{t}^{\alpha} V\left(x_{i}(t)\right)=\sum_{i}^{n} \omega_{i 0}^{C} D_{t}^{\alpha} \phi_{i}\left(x_{i}(t)\right) .
$$

Now, consider the Lyapunov function

$$
V=\omega_{1} e_{h}+\omega_{2} i_{h}+\omega_{3} e_{m}+\omega_{4} i_{m} .
$$

The Caputo derivative of $V$ is then given by

$$
{ }_{0}^{C} D_{t}^{\alpha} V=\omega_{10}^{C} D_{t}^{\alpha} e_{h}+\omega_{20}^{C} D_{t}^{\alpha} i_{h}+\omega_{30}^{C} D_{t}^{\alpha} e_{m}+\omega_{40}{ }^{C} D_{t}^{\alpha} i_{m} .
$$

From the model system (9a)-(9f), we have

$$
\begin{aligned}
{ }_{0}^{C} D_{t}^{\alpha} V= & \omega_{1}\left[\lambda_{m h} i_{m} s_{h}-\left(\varepsilon_{h}+\mu_{h}\right) e_{h}\right]+\omega_{2}\left[\varepsilon_{h} e_{h}-\left(\mu_{h}+\gamma_{h}+d\right) i_{h}\right] \\
& +\omega_{3}\left[\lambda_{h m} i_{h} s_{m}-\left(\varepsilon_{m}+\mu_{m}\right) e_{m}\right]+\omega_{4}\left[\varepsilon_{m} e_{m}-\left(\mu_{m}-b_{m} q\right) i_{m}\right] \\
\leq & \omega_{1}\left[\lambda_{m h} i_{m} s_{h}^{*}-\left(\varepsilon_{h}+\mu_{h}\right) e_{h}\right]+\omega_{2}\left[\varepsilon_{h} e_{h}-\left(\mu_{h}+\gamma_{h}+d\right) i_{h}\right] \\
& +\omega_{3}\left[\lambda_{h m} i_{h} s_{m}^{*}-\left(\varepsilon_{m}+\mu_{m}\right) e_{m}\right]+\omega_{4}\left[\varepsilon_{m} e_{m}-\left(\mu_{m}-b_{m} q\right) i_{m}\right] .
\end{aligned}
$$

If we choose $\omega_{1}=\varepsilon_{h}, \omega_{2}=\varepsilon_{h}+\mu_{h}, \omega_{3}=\varepsilon_{m}, \omega_{4}=\varepsilon_{m}+\mu_{m}$ and simplify the equation, we have

$$
\begin{aligned}
{ }_{0}^{C} D_{t}^{\alpha} V & \leq \varepsilon_{h} \lambda_{m h} i_{m} s_{h}^{*}-\left(\varepsilon_{h}+\mu_{h}\right)\left(\mu_{h}+\gamma_{h}+d\right) i_{h}+\varepsilon_{m} \lambda_{h m} i_{h} s_{m}^{*}-\left(\varepsilon_{m}+\mu_{m}\right)\left(\mu_{m}-b_{m} q\right) i_{m} \\
& =-\left(\varepsilon_{h}+\mu_{h}\right)\left(\mu_{h}+\gamma_{h}+d\right) i_{h}\left(1-\frac{\varepsilon_{m} \lambda_{h m} s_{m}^{*}}{\left(\varepsilon_{h}+\mu_{h}\right)\left(\mu_{h}+\gamma_{h}+d\right)}\right)-\left(\varepsilon_{m}+\mu_{m}\right)\left(\mu_{m}-b_{m} q\right) i_{m}\left(1-\frac{\varepsilon_{h} \lambda_{m h} s_{h}^{*}}{\left(\varepsilon_{m}+\mu_{m}\right)\left(\mu_{m}-b_{m} q\right)}\right) .
\end{aligned}
$$


At disease-free equilibrium, $s_{h}^{*}=\left(b_{h} / \mu_{h}\right)$ and $s_{m}^{*}=\left(b_{m} / \mu_{m}\right)$. Therefore, we have

$$
\begin{aligned}
{ }_{0}^{C} D_{t}^{\alpha} V & \leq-\left(\varepsilon_{h}+\mu_{h}\right)\left(\mu_{h}+\gamma_{h}+d\right) i_{h}\left(1-\frac{\varepsilon_{m} \lambda_{h m} b_{m}}{\left(\varepsilon_{h}+\mu_{h}\right)\left(\mu_{h}+\gamma_{h}+d\right) \mu_{m}}\right)-\left(\varepsilon_{m}+\mu_{m}\right)\left(\mu_{m}-b_{m} q\right) i_{m}\left(1-\frac{\varepsilon_{h} \lambda_{m h} b_{h}}{\left(\varepsilon_{m}+\mu_{m}\right)\left(\mu_{m}-b_{m} q\right) \mu_{h}}\right) \\
& =-\left(\varepsilon_{h}+\mu_{h}\right)\left(\mu_{h}+\gamma_{h}+d\right) i_{h}\left(1-R_{h m}\right)-\left(\varepsilon_{m}+\mu_{m}\right)\left(\mu_{m}-b_{m} q\right) i_{m}\left(1-R_{m h}\right),
\end{aligned}
$$

where $R_{h m}=\left(\varepsilon_{h} \lambda_{m h} b_{h} /\left(\varepsilon_{h}+\mu_{h}\right)\left(\mu_{h}+\gamma_{h}+d\right) \mu_{h}\right)$ is the mean number of the infected mosquitoes due to one infected human and $R_{m h}=\left(\varepsilon_{h} \lambda_{m h} b_{h} /\left(\varepsilon_{m}+\mu_{m}\right)\left(\mu_{m}-b_{m} q\right) \mu_{h}\right)$ is the mean number of infected human due one infected mosquito. Observe that the product of $R_{m h}$ and $R_{h m}$ gives $\mathscr{R}_{0}^{2}$ which is less than 1 at disease-free equilibrium. Consequently, $R_{m h}$ and $R_{h m}$ are less than, and therefore we have ${ }_{0}^{C} D_{t}^{\alpha} V \leq 0$. Thus, the disease-free equilibrium is globally asymptotically stable at $\mathscr{R}_{0}<1$.

3.3. Global Stability of the Endemic Equilibrium. In section 3.1, we established that the disease-free equilibrium is locally stable. The local stability of the disease-free equilibrium implies local stability of the endemic equilibrium in counterpart [21]. Therefore, we only investigate the global stability of the endemic equilibrium by Lyapunov functional approach.

From Definition 3, define $\phi_{i}\left(x_{i}(t)\right)$ by $\phi_{i}\left(x_{i}(t)\right)=x_{i}(t)-x_{i}^{*}-x_{i}^{*} \ln \left(x_{i}(t) / x_{i}^{*}\right)$ where $x^{*}$ is the equilibrium point of the model systems (9a)-(9f). Then, we have the following corollary as in the study by Bourkhouima et al. [10].

Corollary 1. Let $x(t) \in \mathbb{R}^{+}$be a continuous differentiable function. Then, for any $t \geq 0, \alpha \in(0,1)$ and $x^{*} \geq 0$, we have

$$
\begin{aligned}
{ }_{0}^{C} D_{t}^{\alpha} V\left(x_{i}(t)\right) & =\sum_{i}^{n}{ }_{0}^{C} D_{t}^{\alpha}\left(x_{i}(t)-x_{i}^{*}-x_{i}^{*} \ln \frac{x_{i}(t)}{x_{i}^{*}}\right) \\
& \leq \sum_{i}^{n} \omega_{i}\left(1-\frac{x_{i}^{*}}{x_{i}(t)}\right){ }_{0}^{C} D_{t}^{\alpha} x_{i}(t) .
\end{aligned}
$$

Now, using Definition 3 and Corollary 1, we consider the Lyapunov function

$$
\begin{aligned}
V= & \omega_{1}\left(s_{h}-s_{h}^{*}-s_{h}^{*} \ln \frac{s_{h}}{s_{h}^{*}}\right)+\omega_{2}\left(e_{h}-e_{h}^{*}-e_{h}^{*} \ln \frac{e_{h}}{e_{h}^{*}}\right)+\omega_{3}\left(i_{h}-i_{h}^{*}-i_{h}^{*} \ln \frac{i_{h}}{i_{h}^{*}}\right)+\omega_{4}\left(s_{m}-s_{m}^{*}-s_{m}^{*} \ln \frac{s_{m}}{s_{m}^{*}}\right) \\
& +\omega_{5}\left(e_{m}-e_{m}^{*}-e_{m}^{*} \ln \frac{e_{m}}{e_{m}^{*}}\right)+\omega_{6}\left(i_{m}-i_{m}^{*}-i_{m}^{*} \ln \frac{i_{m}}{i_{m}^{*}}\right) .
\end{aligned}
$$

The Caputo derivative of $V$ is then given by

$$
\begin{aligned}
{ }_{0}^{C} D_{t}^{\alpha} V \leq & \omega_{1}\left(1-\frac{s_{h}^{*}}{s_{h}}\right)_{0}^{C} D_{t}^{\alpha} s_{h}+\omega_{2}\left(1-\frac{e_{h}^{*}}{e_{h}}\right)_{0}^{C} D_{t}^{\alpha} e_{h}+\omega_{3}\left(1-\frac{i_{h}^{*}}{i_{h}}\right)_{0}^{C} D_{t}^{\alpha} i_{h}+\omega_{4}\left(1-\frac{s_{m}^{*}}{s_{m}}\right)_{0}^{C} D_{t}^{\alpha} s_{m}+\omega_{5}\left(1-\frac{e_{m}^{*}}{e_{m}}\right)_{0}^{C} D_{t}^{\alpha} e_{m} \\
& +\omega_{6}\left(1-\frac{i_{m}^{*}}{i_{m}}\right)_{0}^{C} D_{t}^{\alpha} i_{m} .
\end{aligned}
$$


From the model systems (9a)-(9f), we have

$$
\begin{aligned}
{ }_{0}^{C} D_{t}^{\alpha} V \leq & \omega_{1}\left(1-\frac{s_{h}^{*}}{s_{h}}\right)\left[b_{h}-\mu_{h} s_{h}-\lambda_{m h} i_{m} s_{h}\right]+\omega_{2}\left(1-\frac{e_{h}^{*}}{e_{h}}\right)\left[\lambda_{m h} i_{m} s_{h}-\left(\varepsilon_{h}+\mu_{h}\right) e_{h}\right] \\
& +\omega_{3}\left(1-\frac{i_{h}^{*}}{i_{h}}\right)\left[\varepsilon_{h} e_{h}-\left(\mu_{h}+\gamma_{h}+d\right) i_{h}\right]+\omega_{4}\left(1-\frac{s_{m}^{*}}{s_{m}}\right)\left[b_{m}\left(1-q i_{m}\right)-\mu_{m} s_{m}-\lambda_{h m} i_{h} s_{m}\right] \\
& +\omega_{5}\left(1-\frac{e_{m}^{*}}{e_{m}}\right)\left[\lambda_{h m} i_{h} s_{m}-\left(\varepsilon_{m}+\mu_{m}\right) e_{m}\right]+\omega_{6}\left(1-\frac{i_{m}^{*}}{i_{m}}\right)\left[\varepsilon_{m} e_{m}-\left(\mu_{m}-b_{m} q\right) i_{m}\right] .
\end{aligned}
$$

Further simplification gives where $P=\left(s_{h}, e_{h}, i_{h}, s_{m}, e_{m}, i_{m}\right) \geq 0$ and

${ }_{0}^{C} D_{t}^{\alpha} V \leq-\omega_{1} \mu_{h} s_{h}\left(1-\frac{s_{h}^{*}}{s_{h}}\right)^{2}-\omega_{4} \mu_{m} s_{m}\left(1-\frac{s_{m}^{*}}{s_{m}}\right)^{2}+F(P)$,

$$
\begin{aligned}
F(P)= & \omega_{1}\left(1-\frac{s_{h}^{*}}{s_{h}}\right)\left(1-\frac{i_{m} s_{h}}{i_{m}^{*} s_{h}^{*}}\right) \lambda_{m h} i_{m}^{*} s_{h}^{*}+\omega_{2}\left(1-\frac{e_{h}^{*}}{e_{h}}\right)\left(\frac{i_{m} s_{h}}{i_{m}^{*} s_{h}^{*}}-\frac{e_{h}}{e_{h}^{*}}\right) \lambda_{m h} i_{m}^{*} s_{h}^{*} \\
& +\omega_{3}\left(1-\frac{i_{h}^{*}}{i_{h}}\right)\left(\frac{e_{h}}{e_{h}^{*}}-\frac{i_{h}}{i_{h}^{*}}\right) \varepsilon_{h} e_{h}^{*}+\omega_{4}\left(1-\frac{s_{m}^{*}}{s_{m}}\right)\left(1-\frac{i_{m}}{i_{m}^{*}}\right) b_{m} q i_{m}^{*} \\
& +\omega_{4}\left(1-\frac{s_{m}^{*}}{s_{m}}\right)\left(1-\frac{i_{h} s_{m}}{i_{h}^{*} s_{m}^{*}}\right) \lambda_{h m} i_{h}^{*} s_{m}^{*}+\omega_{5}\left(1-\frac{e_{m}^{*}}{e_{m}}\right)\left(\frac{i_{h} s_{m}}{i_{h}^{*} s_{m}^{*}}-\frac{e_{m}}{e_{m}^{*}}\right) \lambda_{h m} i_{h}^{*} s_{m}^{*} \\
& +\omega_{6}\left(1-\frac{i_{m}^{*}}{i_{m}}\right)\left(\frac{e_{m}}{e_{m}^{*}}-\frac{i_{m}}{i_{m}^{*}}\right) \varepsilon_{m} e_{m}^{*} .
\end{aligned}
$$

If we choose and perform further simplification, we have

$$
\begin{aligned}
& \omega_{1}=\omega_{2}=\frac{1}{\lambda_{m h} i_{m}^{*} s_{h}^{*}}, \\
& \omega_{3}=\frac{1}{\varepsilon_{h} e_{h}^{*}}, \\
& \omega_{4}=\omega_{5}=\frac{1}{\lambda_{h m} i_{h}^{*} s_{m}^{*}}, \\
& \omega_{5}=\frac{1}{\varepsilon_{m} e_{m}^{*}},
\end{aligned}
$$

$$
F(P)=\left(3-\frac{s_{h}^{*}}{s_{h}}-\frac{i_{h}^{*} e_{h}}{i_{h} e_{h}^{*}}-\frac{e_{h}^{*} i_{m} s_{h}}{e_{h} i_{m}^{*} s_{h}^{*}}\right)+\left(3-\frac{s_{m}^{*}}{s_{m}}-\frac{i_{m}^{*} e_{m}}{i_{m} e_{m}^{*}}-\frac{e_{m}^{*} i_{h} s_{m}}{e_{m} i_{h}^{*} s_{m}^{*}}\right)+\left(1-\frac{s_{m}^{*}}{s_{m}}\right)\left(1-\frac{i_{m}}{i_{m}^{*}}\right) \frac{b_{m} q i_{m}^{*}}{\lambda_{h m} i_{h}^{*} s_{m}^{*}} .
$$


TABle 2: Parameters and their description.

\begin{tabular}{|c|c|c|c|}
\hline Parameter & Description & Value per day & Source \\
\hline$b_{h}$ & Birth rate in human & 0.0015 & [8] \\
\hline$b_{m}^{n}$ & Birth rate in mosquitoes & 0.01 & Estimated \\
\hline$\lambda_{h m}^{m}$ & Adequate contact rate: human to mosquito & 0.375 & [7] \\
\hline$\lambda_{m h}$ & Adequate contact rate: mosquito to human & 0.245 & Estimated \\
\hline$\varepsilon_{m} n$ & $1 /$ (incubation period of mosquitoes in days) & $1 / 8$ & [9] \\
\hline$\varepsilon_{h}$ & $1 /$ (incubation period of human in days) & $1 / 6$ & [9] \\
\hline$\mu_{m}$ & $1 /($ life span of mosquitoes in days) & $1 / 60$ & [9] \\
\hline$\mu_{h}$ & Natural death rate in human & 0.000042 & {$[23]$} \\
\hline$q$ & Rate of vertical transmission & 0.05 & {$[8]$} \\
\hline$d$ & Disease-induced death rate in human & 0.01 & {$[7]$} \\
\hline$\gamma_{h}$ & $1 /$ (infectious period of human in days) & $1 / 7$ & [9] \\
\hline
\end{tabular}

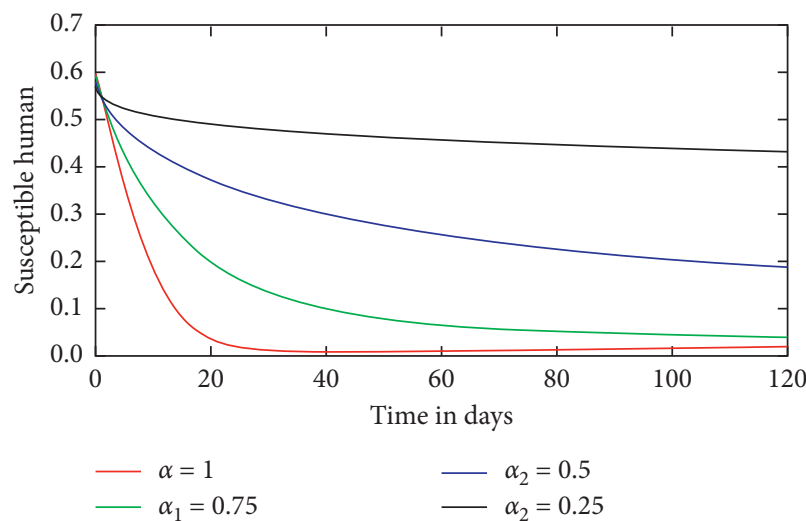

(a)

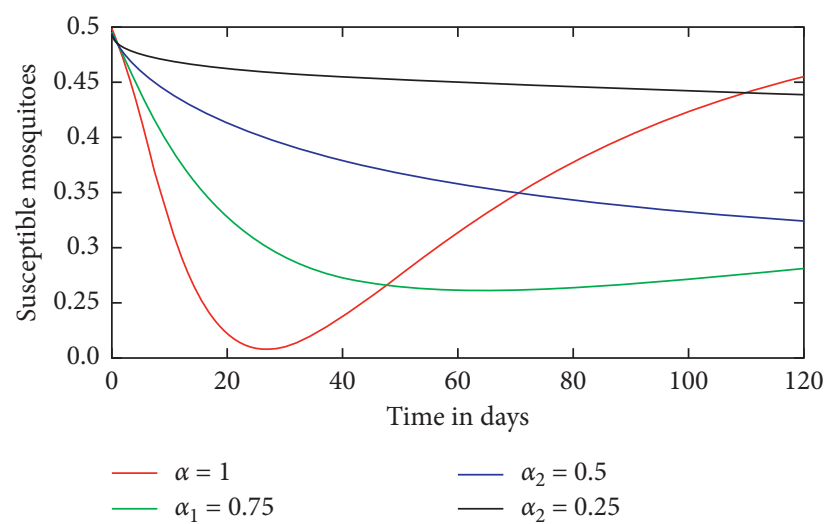

(b)

FIGURE 2: Variations of susceptible population at $\alpha=1,0.75,0.50$, and 0.25: (a) susceptible human; (b) susceptible mosquitoes.

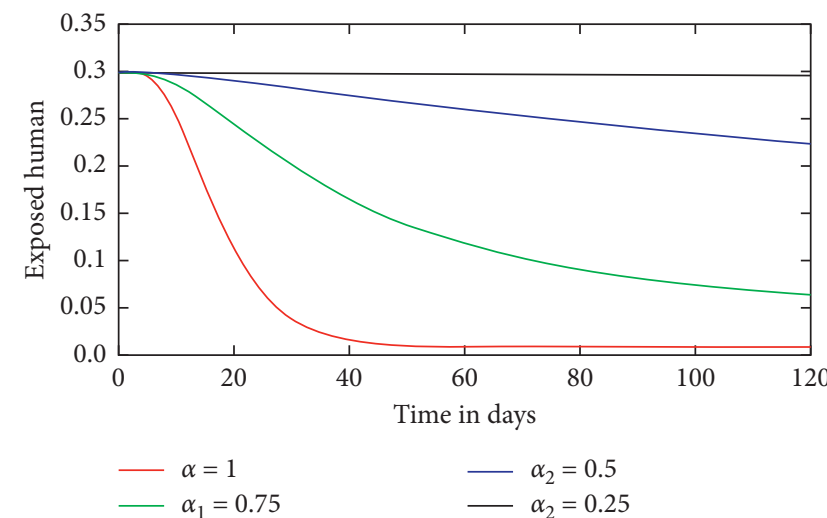

(a)

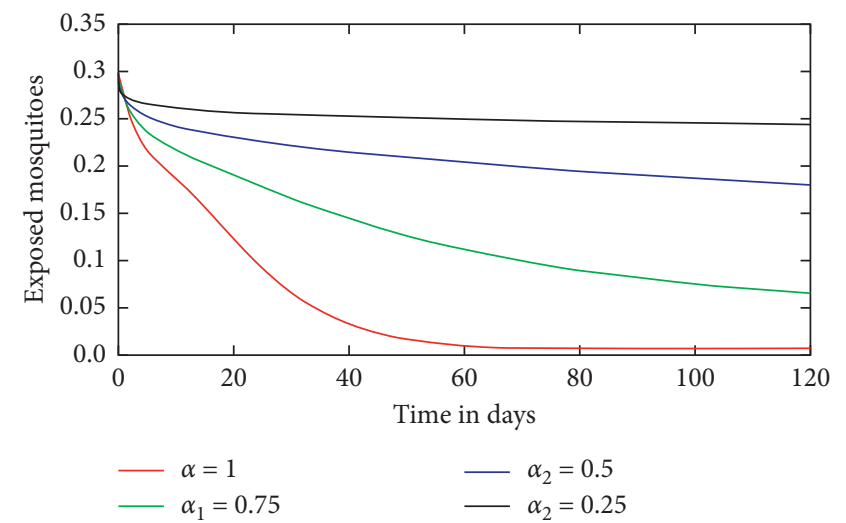

(b)

Figure 3: Variations of exposed population at $\alpha=1,0.75,0.50$, and 0.25: (a) exposed human; (b) exposed mosquitoes.

In general, $\left(1-\left(x^{*} / x\right)\right) \geq 0$ and $\left(1-\left(x / x^{*}\right)\right) \leq 0$ if the $x^{*} \quad$ is the equilibrium point. Therefore, $\left(1-\left(s_{m}^{*} / s_{m}\right)\right)\left(1-\left(i_{m} / i_{m}^{*}\right)\right) \leq 0$, and hence $F(P)$ is nonpositive in $P$. Thus, ${ }_{0}^{C} D_{t}^{\alpha} V \leq 0$ in $P$ and is zero when $P=P^{*}$. Following LaSalle's invariant principle, it is concluded that $P^{*}$ is globally asymptotically stable.

\section{Numerical Simulations}

This section presents results from numerical simulations for the model system (9a)-(9f). The simulation is done using the predictor-corrector method implemented in Matlab fde12 [22]. The initial values used in simulations are 


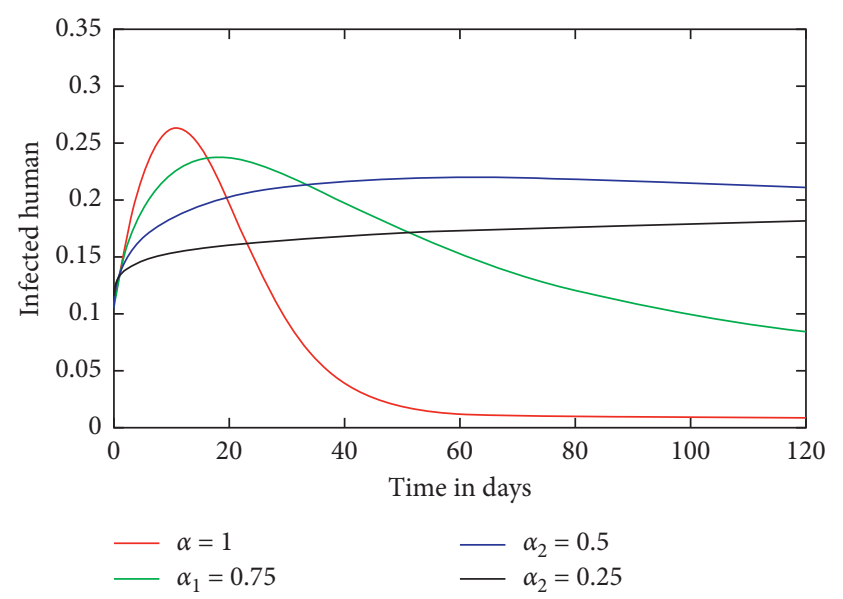

(a)

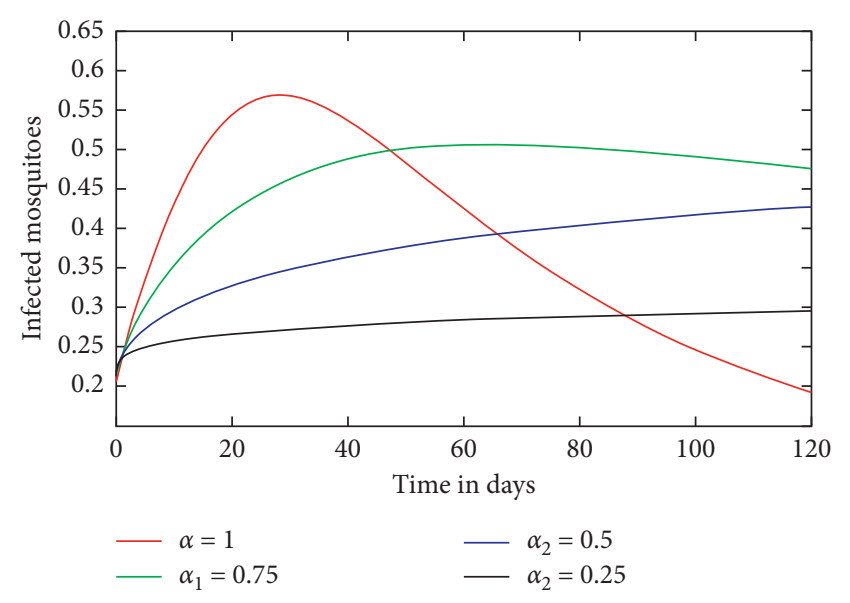

(b)

Figure 4: Variations of infected population at $\alpha=1,0.75,0.50$, and 0.25: (a) infected human; (b) infected mosquitoes.

$s_{h}=0.6, e_{h}=0.3, i_{h}=0.1, s_{m}=0.5, e_{m}=0.3$, and $i_{m}=0.2$. The mosquito birth rate $b_{h}$ has been estimated from the number of eggs laid per day, and the adequate contact rate from mosquito to human $\lambda_{m h}$ has been estimated from the biting rate and the probability that a mosquito can bite human and cause infection. Other parameter values and their source are as given in Table 2.

Figure 2 shows the variations of susceptible human and mosquito animal population. In Figure 2(a), the human susceptible population decreases with increase in the order $\alpha$, while, in Figure 2(b), there is a rapid increase in susceptible mosquitoes just after the first 30 days for order $\alpha=1$, surpassing all orders.

Figure 3 shows the variations of exposed human and mosquito animal population. In Figure 3(a), the human exposed population decreases with increase in the order $\alpha$, where a similar trend is shown also in Figure 3(b) of exposed mosquitoes.

Figure 4 shows the variations of infected human and mosquito animal population. In Figure 4(a), the human infected population decreases with the decrease in the order $\alpha$. However, there is a rapid decrease for $\alpha=1$ and $\alpha=0.75$ towards the disease-free equilibrium. In Figure 2(b), a rapid decrease in infected mosquitoes is seen for the order $\alpha=1$, surpassing all orders.

\section{Discussion}

In this paper, a fractional-order derivative approach using Caputo derivative has been used to investigate the dynamics of RVF in an urban peridomestic cycle. The basic reproduction number $\mathscr{R}_{0}$ of the model was computed to study the effect of initial transmission of the disease and used to analyse the stability of the disease-free equilibrium and endemic equilibrium. The analysis of the stability of equilibrium points indicated that both the disease-free equilibrium and endemic equilibrium of the model system are locally and globally asymptotically stable. The stability of the disease-free equilibrium means that the RVF outbreak can be controlled provided that $\mathscr{R}_{0}<1$.
Numerical simulations were performed to analyse the population variation with respect to the time and order $\alpha=1,0.75,0.5$, and 0.25 . In general, the results from simulations show that whenever there is an increase in susceptible and exposed population in both human and mosquitoes, the value of $\alpha$ decreases. A rapid increase in susceptible mosquitoes is observed just after the first 30 days for order $\alpha=1$, surpassing all orders. The infected population decreases with a decrease in the value of $\alpha$. However, there is a rapid decrease in infected human and mosquitoes after the first 30 days for the order $\alpha=1$, surpassing all orders.

\section{Conclusion}

Rift Valley fever will remain endemic in many countries in the world because of its dynamical behaviour and RVF virus maintenance during the period of no outbreak in the endemic areas. Its ability to emerge and re-emerge after several years makes RVF infection a disease of global concern. The ability of the RVF viruses to adopt transmission cycles involving different mosquito species poses a greater risk in human health and poses need to looking into the social and environmental factors which favour the growth of mosquito vectors in urban areas. The results of the analysis show that the disease is persistent when introduced in the society causing threat to human health. Effective educational campaign about the transmission dynamics of RVF and proper hygiene may help to reduce the infection.

\section{Data Availability}

The set of parameter values are mainly from articles similar to the work, while the unavailable data especially values of parameter were estimated for the purpose of verifying results of the mathematical analysis of the model developed.

\section{Conflicts of Interest}

The author declares there are no conflicts of interest regarding the publication of this manuscript. 


\section{References}

[1] R. Swanepoel and J. T. Paweska, "Rift Valley fever," in Oxford Textbook of Zoonoses: Biology, Clinical Practice, and Public Health Control, S. R. Palmer, L. Soulsby, P. R. Torgerson, and D. W. G. Brown, Eds., pp. 421-431, Oxford University Press, Oxford, UK, 2011.

[2] R. Daubney, J. R. Hudson, and P. C. Garnham, "Enzootic hepatitis or rift valley fever. An undescribed virus disease of sheep cattle and man from east Africa," The Journal of $\mathrm{Pa}$ thology and Bacteriology, vol. 34, no. 4, pp. 545-579, 1931.

[3] J. T. Paweska, "Rift valley fever," Revue Scientifique et Technique de l'OIE, vol. 34, no. 2, pp. 375-389, 2015.

[4] G. H. Gerdes, "Rift valley fever," Revue Scientifique et Technique de l'OIE, vol. 23, no. 2, pp. 613-623, 2004.

[5] A. Ahmed, Y. Ali, A. Elduma et al., "Unique outbreak of Rift valley fever in Sudan, 2019," Emerging Infectious Diseases, vol. 26, no. 12, pp. 3030-3033, 2020.

[6] R. Métras, W. J. Edmunds, C. Youssouffi et al., "Estimation of Rift valley fever virus spillover to humans during the Mayotte 2018-2019 epidemic," Proceedings of the National Academy of Sciences, vol. 117, no. 39, pp. 24567-24574, 2020.

[7] S. C. Mpeshe, H. Haario, and J. M. Tchuenche, "Amathematical model of Rift valley fever with human host," Acta Biotheoretica, vol. 59, no. 3-4, pp. 231-250, 2011.

[8] S. C. Mpeshe, L. S. Luboobi, and Y. Nkansah-Gyekye, "Modeling the impact of climate change on the dynamics of Rift valley fever," Computational and Mathematical Methods in Medicine, vol. 2014, Article ID 627586, 12 pages, 2014.

[9] S. C. Mpeshe, L. S. Luboobi, and Y. Nkasah-Gyekye, "Stability analysis of the Rift valley fever dynamical model," Journal of Mathematics and Computer Science, vol. 4, no. 4, pp. 740-762, 2014.

[10] A. Boukhouima, K. Hattaf, E. M. Lotfi, M. Mahrouf, D. F. M. Torres, and N. Yousfi, "Lyapunov functions for fractional-order systems in biology: methods and applications, chaos," Solitons \& Fractals, vol. 140, 2020.

[11] C. J. Silva and D. F. M. Torres, "Stability of a fractional HIV/ AIDS model," Mathematics and Computers in Simulation, vol. 164, pp. 180-190, 2019.

[12] B. Ghanbari, H. Gunerhan, and H. M. Srivastava, "An application of the Atangana-Baleanu fractional derivative in mathematical biology: a three-species predator-prey model," Chaos Solitons Fractals, vol. 138, 2020.

[13] B. S. T. Alkahtani and S. S. Alzaid, "A novel mathematics model of Covid-19 with fractional derivative. Stability and numerical analysis," Chaos, Solitons and Fractals, vol. 138, pp. 1-11, 2020.

[14] N. H. Tuan, H. Mohammadi, and S. Rezapour, "A mathematical model for COVID-19 transmission by using the Caputo fractional derivative," Chaos, Solitons and Fractals, vol. 140, pp. 1-11, 2020.

[15] M. A. Khan and A. Atangana, "Modeling the dynamics of novel coronavirus (2019-nCov) with fractional derivative," Alexandria Engineering Journal, vol. 59, no. 4, pp. 2379-2389, 2020.

[16] F. Ndaïrou, I. Area, J. J. Nieto, C. J. Silva, and D. F. M. Torres, "Fractional model of COVID-19 applied to Galicia, Spain and Portugal," Chaos, Solitons and Fractals, vol. 144, 2021.

[17] M. A. Aba Oud, A. Ali, H. Alrabaiah, S. Ullah, M. A. Khan, and S. Islam, "A fractional order mathematical model for COVID-19 dynamics with quarantine, isolation, and environmental viral load," Advances in Difference Equations, vol. 2021, no. 1, 2021.
[18] Y.-M. Chu, A. Ali, M. A. Khan, S. Islam, and S. Ullah, "Dynamics of fractional order COVID-19 model with a case study of Saudi Arabia," Results in Physics, vol. 21, 2021.

[19] K. Diethelm, N. J. Ford, and A. D. Freed, "A predictor-corrector approach for the numerical solution of fractional differential equations," Nonlinear Dynamics, vol. 29, no. 1/4, pp. 3-22, 2002.

[20] I. Petras, "Fractional-order nonlinear systems: modeling, analysis and simulation," Series: Nonlinear Physical Science, Springer, Berlin, Germany, 2011.

[21] P. van den Driessche and J. Watmough, "Reproduction numbers and sub-threshold endemic equilibria for compartmental models of disease transmission," Mathematical Biosciences, vol. 180, no. 1-2, pp. 29-48, 2002.

[22] R. Garrappa, Predictor-Corrector PECE Method for Fractional Differential Equations, MATLAB Central, Natick, MA, USA, 2020, https://www.mathworks.com/matlabcentral/ fileexchange/32918-predictor-corrector-pece-methodforfractional- differential- equations.

[23] S. C. Mpeshe and N. Nyerere, "Modeling the dynamics of coronavirus disease pandemic coupled with fear epidemics," Computational and Mathematical Methods in Medicine, vol. 2021, Article ID 6647425, 9 pages, 2021. 